WORK IN PROGRESS

\title{
Towards development of an effective low-cost interactive whiteboard for promoting student engagement in sociology courses
}

\author{
Miguel Garcia-Ruiz, Vivian Jimenez Estrada, \\ Deborah Woodman
}

Published: 21 September 2016

\begin{abstract}
Interactive whiteboards (IWBs) can be a great asset to teachers trying to help students visualize what they are trying to teach, especially for supporting instructors and teams of students in class. However, commercial IWBs can be costly and difficult to operate. This paper describes the development of a low-cost IWB using a video game controller and "homebrew" infrared pens, and how its usability and technology acceptance will be tested in the context of sociology courses. We propose that the use of low-cost and highly-usable technologies in sociology courses may enhance student learning outcomes and retention rates as a way to fit the learning needs of students and supporting them in the engagement of critical thinking.
\end{abstract}

Keywords: Interactive whiteboard, iterative development, usability, technology acceptance, sociology education, student engagement.

\section{Introduction}

Interactive whiteboards, or IWBs (large interactive wall-mounted displays connected to a computer, where a user can control it using a stylus and other devices by touching the display) can be a great asset to teachers trying to help students visualize what they are trying to teach - whether it be graph theory, diagram, or any other subject, making an otherwise static lesson dynamic. It should keep students engaged, and helps to quickly get a complex point across. In addition, an IWB could foster collaboration among student team members in class [1].

However, commercial IWBs can be costly, some of them are difficult to install, they require proprietary commercial software, and sometimes are difficult to maintain and apply [1]. A much cheaper, adaptable and nearly comparable solution can be implemented for a fraction of the cost, such as in [2]. Using a Nintendo Wii video game controller ("Wiimote"), a conventional data projector, and an infrared pen (it can be easily built in-

Garcia-Ruiz, M., Jimenez Estrada, V., Woodman, D.

Algoma University

1520 Queen St E,

Sault Ste. Marie, Canada

Email: miguel.garcia@algomau.ca,vivian.estrada@algomau.ca,

deborah.woodman@algomau.ca house), an instructor and/or students can interact with an IWB using the natural control of freehand writing without having to remain behind the desktop computer [2].

Past literature (e.g. [1]) reports that IWBs have been successfully used in classrooms for many years, fostering active learning, as well as enhancing active social participation of both students and teachers who use the IWBs. This research will further investigate if simple technologies such as low-cost IWBs have the potential to engage students in the social sciences, and promote exchanges of ideas that support the social construction of knowledge. The literature also shows that student engagement is an area that professors are focusing on, particularly with seminal works that highlight the "death of the lecture", further suggesting that work in the area of student engagement is key to retaining students and increase completion rates [3]. According to Government of Canada's Highly Skilled Workforce Report (https://www.ontario.ca/page/building-workforce-tomorrow-

shared-responsibility), the implementation of this technology can potentially "ensure adult learners have access to quality experiential learning opportunities that are adaptable and appropriate to their needs." (P. 2) To this end, we will implement a project that will find out the acceptability and potential impact IWBs have on retention and engagement rates for students in Sociology courses at Algoma University Canada. The purpose of this poster is twofold: To describe the development of a low-cost IWB and proposed usability and technology acceptance testing of the IWB within the context of sociology courses.

\section{Whiteboard development}

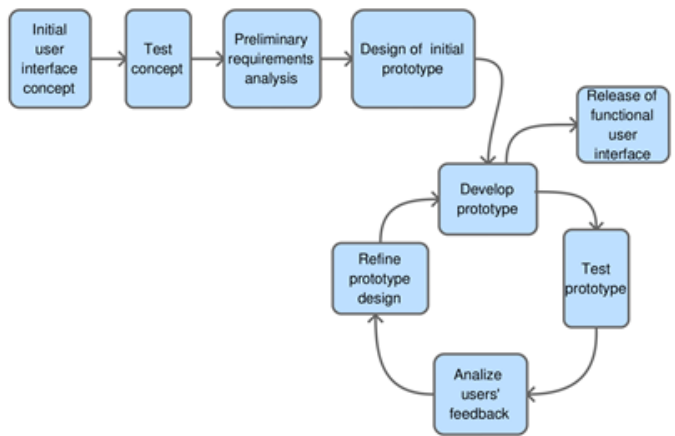

Figure 1. Our Evolutionary Prototyping lifecycle model 
A computer science student and the first author developed and tested iteratively a software and hardware-based prototype of an IWB based on [2]. It has been developed following the Evolutionary Prototyping lifecycle model and taking into account the user centered design (UCD) paradigm [4]. In addition, our method oversees the importance of prototype evaluation. Initial evaluation tasks included to freely draw diagrams and geometric figures on the IWB. Figure 1 shows a diagram of the evolutionary prototyping method, which we adapted from [5].

\section{The interactive whiteboard}

Figure 2 shows a student testing the developed IWB. The Wiimote was placed about three meters from the projection screen. The data projector (no shown in the figure) is hanging from the classroom ceiling. The hardware is fairly easy to set up.

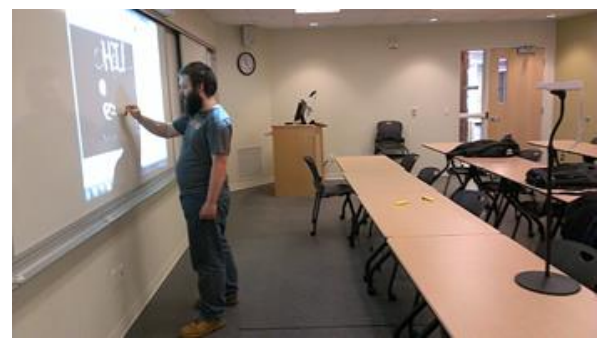

Figure 2. A student testing the developed whiteboard

Figure 3 shows three infrared pens that we built using stationery markers and some low-cost electronic components (a $1.5 \mathrm{~V}$ battery, a $10 \mathrm{Ohm}$ resistor, a push button and an infrared LED) and an electronic diagram for making the infrared pen. The figure also shows the Wiimote controller used in the IWB and the USB Bluetooth dongle. The IWB can be built under \$80 USD not including the host computer and the data projector.

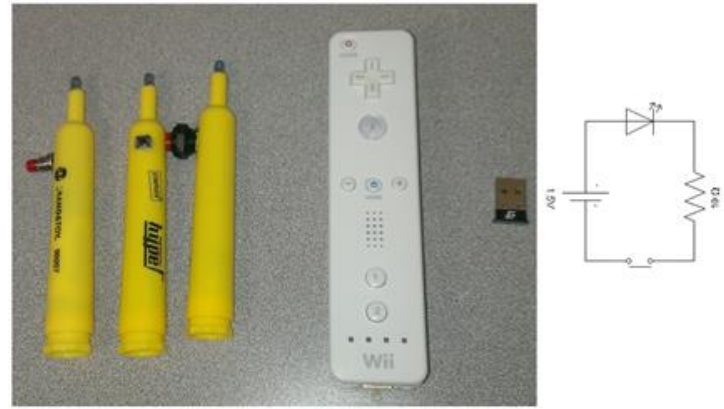

Figure 3. The infrared pens, Wiimote and Bluetooth dongle

A computer science student built the infrared pens (shown in Figure 3) and programmed the necessary software for controlling the IWB. Figure 2 shows the Wiimote placed at the right on a stand, which reads the position of the infrared pen. The Wiimote is connected via Bluetooth to the host desktop computer that had the Bluetooth dongle connected, shown at the center in Figure 2. The student adapted and programmed the software necessary for running the IWB, which is easy to install. The original software version was downloaded freely from: http://johnnylee.net/projects/wii/.

\section{Further whiteboard testing}

We plan to conduct formative user studies such as interviews and the Think Aloud Protocol for assessing the whiteboard usability and later do instructional design and plan learning experiences using our fully-fledged IWB. We also plan to assess the user acceptance of our IWB by using the Technology Acceptance Model Version 2 questionnaire [6], including questions on user motivation and engagement, technology effectiveness and perceived usefulness. We will include sociology students and professors from Algoma University in all the testing, and we will try to answer the following research questions with our study outcomes: To what extent do students' acceptance of IWB in higher education promote their usability, student motivation and technology acceptance? What are the impacts on student engagement and retention rates in a sociology course?

\section{Conclusions}

This poster proposal described the development of a low-cost interactive whiteboard (IWB) based on [2] using the Evolutionary Prototyping and UCD models. We propose that the use of lowcost and highly-usable technologies in sociology courses may enhance student learning outcomes and retention rates as a way to fit the learning needs of students. This will be studied with our IWB. This proposition promises to find some solutions to connecting students to the curriculum and this enhance student engagement and critical thinking.

\section{Acknowledgments}

This research is supported by the Algoma University Research Fund (AURF).

\section{References}

[1] Lacina, J. 2009. Technology in the classroom interactive whiteboards: Creating higher-level, technological Thinkers?. Childhood Education, 85(4), 270-272.

[2] Lee, j.C. 2008. Hacking the Nintendo Wii remote. IEEE Pervasive Computing, July-September, 39-45.

[3] Englund, C., Olofsson, A.D. and Price, L.. 2016. Teaching with technology in higher education: understanding conceptual change and development in practice. Higher Education Research \& Development, April issue.

[4] Lowdermilk, T. 2012. User-centered design. O'reilly,

[5] Sebastopol, CA.

[6] McConnell, S. 1996. Rapid development: Taming wild software schedules. Microsoft Press, Redmond, WA.

[7] Venkatesh, V., and Davis, F. D. 2000. A theoretical extension of the technology acceptance model: Four longitudinal field studies. Management science, 46(2), 186204. 